\title{
The role of barley cuticular wax in disease resistance
}

\author{
Rozanova I. ${ }^{1,2 *}$, Gerasimova S. ${ }^{1,2}$, Lasina N. ${ }^{3}$, Afanasenko O. ${ }^{3}$, Khlestkina E. ${ }^{1}$ \\ ${ }^{1}$ Federal Research Center the N.I. Vavilov All-Russian Institute of Plant Genetic Resources (VIR), \\ Saint Petersburg, Russia \\ ${ }^{2}$ Institute of Cytology and Genetics, SB RAS, Novosibirsk, Russia \\ ${ }^{3}$ All-Russian Institute of Plant Protection, Saint Petersburg, Russia \\ *i.rozanova@vir.nw.ru
}

Key words: wax, barley, disease resistance

Motivation and Aim: The improvement of plant protection against biotic stress became a trend in modern agriculture. From one side the resistance is provided by receptor proteins in the plasma membrane, intracellular receptors of the immune response, and loci of quantitative traits. From another side, it can be provided by physical barriers when pathogen spores do not penetrate into the plant. The first physical barrier between plants and pathogens is the cuticle protecting plants against water loss and desiccation, and other abiotic and biotic stresses. The cuticle is composed of a polyester scaffold, cutin, which is covered with cuticular waxes. It was found that there is likely to be interdependence between the microstructure and chemical composition of the host plant surface and the initial signals that trigger the development of pathogenic fungi. The aim of this work is to find out how the presence/absence of certain cuticle wax components affect the barley resistance to fungal diseases.

Methods: Three isogenic mutant lines derived from a double haploid barley line of the Golden Promise variety were taken as a model. All the lines lack ketone wax components at the surface of leaf sheaths. The plants were sown in pots in a phyto room. Infection was carried out with three pathogens: (Cochliolobus sativus), (Pyrenophora teres f. teres) and (Puccinia graminis), causing spot blotch, net blotch and stem rust deseases respectively. Inoculation was carried out by spraying the plants at the stage of the beginning of heading. The innoculated plants were covered with plastic bags and kept at $20-22{ }^{\circ} \mathrm{C}$ for 24 hours in the dark at $100 \%$ relative humidity, then grown at $20-22{ }^{\circ} \mathrm{C}$ and $16 \mathrm{~h}$ day $/ 8 \mathrm{~h}$ night photoperiod at $70 \%$ relative humidity. Accounting for the development of the disease is carried out in the phase of milky-wax ripeness according to the percentage of the affected area of leaves of the upper and lower tiers.

Conclusion: The results obtained in the study help us to understand whether the cuticular wax composition affects plant protection against pathogens.

Acknowledgements: The study is supported by Russian Science Foundation (21-6600012). 